Canadian Journal of Physics

Canadian $\underset{\text { Science Publishing }}{\text { Nachoum }}$

Revue canadienne de physique

\title{
Investigation of the structural and electronic properties of CdS under high pressure: an ab initio study
}

\begin{tabular}{|r|l|}
\hline Journal: & Canadian Journal of Physics \\
\hline Manuscript ID & cjp-2017-0257.R1 \\
\hline Manuscript Type: & Article \\
\hline Date Submitted by the Author: & 20-Jun-2017 \\
\hline Complete List of Authors: & $\begin{array}{l}\text { Yamçıçır, C.; Gazi Universitesi } \\
\text { Merdan, Z.; Gazi Universitesi } \\
\text { Kürkçü, C.; Ahi Evran Universitesi }\end{array}$ \\
\hline $\begin{array}{r}\text { Is the invited manuscript for } \\
\text { consideration in a Special } \\
\text { Issue? : }\end{array}$ & CdS, ab-initio, Intermediate states, Phase transitions, pressure \\
\hline &
\end{tabular}

SCHOLARONE $^{\text {m }}$

Manuscripts 


\title{
Investigation of the structural and electronic properties of CdS under high pressure: an ab initio study
}

\author{
C. Yamcicier ${ }^{\mathrm{a}}$, Z. Merdan ${ }^{\mathrm{b}}$ and C. Kurkcu ${ }^{\mathrm{c}^{*}}$ \\ ${ }^{a}$ Institute of Science, Gazi University, Ankara, Turkey \\ ${ }^{\mathrm{b}}$ Science Faculty, Gazi University, Ankara, Turkey \\ ${ }^{\mathrm{c}}$ Faculty of Arts and Sciences, Ahi Evran University, Kirsehir, Turkey
}

An ab initio constant pressure study is carried out to explore the behaviour of cadmium sulfide $(\mathrm{CdS})$ under high hydrostatic pressure. We have studied the structural properties of CdS using density functional theory (DFT) under pressure up to $200 \mathrm{GPa}$. CdS crystallizes in a wurtzite (WZ)-type structure under ambient conditions. CdS undergoes a structural phase transition from the hexagonal WZ-type structure with space group $\mathrm{P}_{3} \mathrm{mc}$ to cubic NaCl-type structure with space group $\mathrm{Fm} \overline{3} \mathrm{~m}$. Another phase transition is obtained from NaCl-type structure to the orthorhombic CdS-III-type structure with space group Pmmn. The first transformation proceeds via seven intermediate states with space group $\mathrm{Cmc} 2_{1}, \mathrm{P} 2_{1}, \mathrm{Pmn} 2_{1}$, $\mathrm{P} 2{ }_{1} / \mathrm{m}, \mathrm{Pmmn}, \mathrm{I} / \mathrm{mmm}$ and $\mathrm{Cmcm}$. The later transformation is based on two intermediate states with space groups Immm and $\mathrm{P} 2_{1} / \mathrm{m}$. These phase transitions are also studied by total energy and enthalpy calculations. According to these calculations, the phase transformations occur at about $3 \mathrm{GPa}$ and $51 \mathrm{GPa}$, respectively. Calculation results on the other basic properties, such as lattice constant, volume, and bulk modulus are also compared with those of other recent theoretical and experimental data, and generally, good agreement with the available data is obtained.

Keywords: $\mathrm{CdS}, \mathrm{Ab}$-initio, Intermediate states, Phase transitions

\footnotetext{
* Corresponding author. Tel.: +90 386 2804644; fax: +90 3862804525 .

E-mail adresse: ckurkcu@ahievran.edu.tr; (C. Kurkcu).
} 


\section{Introduction}

Cadmium Sulfide (CdS), one of the IIB-VIA semiconductor, has received considerable attention owing to their novel optical and electronic properties used in different technological fields such as solar cells, light emitting diodes, field effect transistors, photoconductors, infrared materials, sensors, energy storage materials and photocatalyst over the past few decades [1-15].

At ambient conditions, CdS has three phases which are hexagonal greenockite wurtzite (WZ)-type structure with space group $\mathrm{P}_{3} \mathrm{mc}$, cubic hawleyite zincblende (ZB)-type structure with space group $F \overline{4} 3 m$ and cubic rocksalt (RS)-type structure with space group $F m \overline{3} m$ [2].

In this paper, we studied the structural and electronic properties of WZ-type structure of CdS under high pressure. The Cadmium (Cd) and Sulfide (S) atoms are located in the same Wyckoff position 2(b) $(1 / 3,2 / 3, \mathrm{z})$ with $\mathrm{z}=0$ and $\mathrm{z}=0,37715$ [11].

Over the recent years, a few groups have investigated phase transition mechanism of CdS using both experimentally $[10,11,17]$ and theoretically $[13,16]$. CdS undergoes a phase transformation from the WZ-type structure with space group $\mathrm{P}_{3} \mathrm{mc}$ to the $\mathrm{NaCl}$-type structure with space group $\mathrm{Fm} \overline{3} \mathrm{~m}$ at about 2-3 GPa $[11,13,16]$. Suziki et al. found that NaCl-type structure of CdS transforms to a new phase called CdS-III at $50 \mathrm{GPa}$ [17].

Benkhettou et al. [10] suggested that this phase has orthorhombic structure with space group Pmmn. Furthermore, Chen et al. [16] founded the CdS-III phase with Pmmn symmetry at above $51 \mathrm{GPa}$ using the ab initio phonon and enthalpy calculations. In the present work, we propose a simulation study of the structural and electronic of CdS. For this material, our results yield the following sequence $\mathrm{P}_{3} \mathrm{mc} \rightarrow \mathrm{Fm} \overline{3} \mathrm{~m} \rightarrow \mathrm{Pmmn}$ which is compatible with the other studies $[11,17]$. Additionally we suggest that the $F m \overline{3} m$ phase of this material proceeds through seven intermediate phase with space group $\mathrm{Cmc} 2_{1}, \mathrm{P} 2_{1}, \mathrm{Pmn} 2_{1}, \mathrm{P} 2_{1} / \mathrm{m}$, Pmmn, I4/mmm and Cmcm expressed as CdS-(a), CdS-(b), CdS-(c), CdS-(d), CdS-(e), CdS(f) and CdS-(g). For the Pmmn phase of CdS, we suggest that it proceeds through two intermediate phases with space group Immm and $\mathrm{P} 2_{1} / \mathrm{m}$ expressed as CdS-(h) and CdS-(i).

Many experiments have been carried out by the researchers for a wide range of crystal systems in order to investigate structural and mechanistic properties. However, the structural and mechanistic phase transformations are not identified clearly due to the limitations of the experimental set ups which left the system unexplained beyond the experimental limitations. In order to overcome these limitations, ab initio method which provides accurate and realistic results is chosen in this study. The phase transition of CdS and the electronic properties of 
different high pressure phases are studied by using the SIESTA code and a simulation study of the transformation mechanism of CdS is proposed.

\section{Method of computation}

All the DFT calculations were performed with Perdew-Burke-Ernzerhof (PBE) generalized gradient approximation (GGA) [18] functional using the ab initio program SIESTA [19]. A conjugate gradient (CG) technique was used for minimization of geometries under a constant pressure. A cutoff energy of 300 Ryd was used in all calculations.

The self-consistent "norm-conserving" pseudopotentials were generated using the Troullier-Martins scheme [20]. In the calculations, double-zeta plus polarized orbitals were used for a real space grid. We used $\Gamma$-point sampling for the Brillouin-zone integration. For the energy-volume calculations, we considered the unit cell for CdS phases. The Brillouinzone integration was performed with $8 \times 8 \times 6,8 \times 8 \times 8$ and $8 \times 6 \times 10$ k-point mesh for $\mathrm{P}_{3} \mathrm{mc}, \mathrm{Fm} \overline{3} \mathrm{~m}$ and Pmmn phases of CdS following the convention of Monkhorst and Pack [21]. We used these k-point meshes for the energy-volume calculations. The simulation cell consist of 72 atoms with periodic boundary conditions. These structures were allowed to relax and to find their equilibrium volumes and lowest energies for each value of the applied pressure by optimizing their lattice vectors and atomic positions. Geometries were relaxed

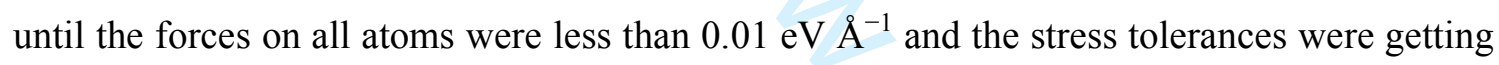
less until $0.5 \mathrm{GPa}$.

Conjugate gradient technique was used to apply external pressure to the system. Pressure was increased with an increment of $5 \mathrm{GPa}$. In order to analyze each minimization step, KPLOT program and RGS algorithm was used $[22,23]$ that gives elaborated knowledge about cell parameters, atomic positions and space group of an analyzed structure. 


\section{Results and discussion}

CdS crystallizes in a hexagonal greenockite WZ-type structure, cubic hawleyite ZBtype structure and cubic RS-type structure at ambient conditions. In this paper, we studied WZ-type structure of CdS under the hydrostatic pressure up to $200 \mathrm{GPa}$. Pressure was gradually increased beginning from the $0 \mathrm{GPa}$ and the structure of $\mathrm{CdS}$ at each applied pressure was analyzed via the KPLOT program. CdS undergoes a phase transformation from the WZ-type structure with space group $\mathrm{P}_{3} \mathrm{mc}$ to the NaCl-type structure with space group $\mathrm{Fm} \overline{3} \mathrm{~m}$ at $10 \mathrm{GPa}$. Another phase transformation from the NaCl-type structure to the CdS-III structure with space group Pmmn was obtained at $165 \mathrm{GPa} . \mathrm{P}_{3} \mathrm{mc}, \mathrm{Fm} \overline{3} \mathrm{~m}$ and Pmmn phases of CdS have 4, 8 and 4 atoms per unit cell, respectively. The crystal structures of these three phase at $0 \mathrm{GPa}, 10 \mathrm{GPa}$ and $165 \mathrm{GPa}$ are depicted in Fig. 1 and its lattice parameters and fractional atomic positions are summarized in Table 1.

We plotted pressure-volume relation and pressure-lattice constants relation to determine the thermodynamic nature of the phase transitions for CdS in Fig. 2. As seen in Fig. 2 , the volume and the lattice constants decrease monotonically and indicate the phase transitions at $10 \mathrm{GPa}$ and $165 \mathrm{GPa}$. It is clear that these discontinuities indicate the first order phase transitions. The reason of the discontinuities can be discussed from the crystal structure variations at high pressure.

Transition pressures obtained in constant-pressure simulations are noticeably larger than the experimental results. This result is anticipated because the simulation conditions such as loading rate, the limited box size, the lack of defects in the simulated structures and the use of periodic boundary conditions are significantly different from the experimental ones. As a result, the systems have to cross a significant energy barrier to transform from one phase to another phase. We then take the energy-volume computations into account so as to study the stability of high pressures phases of CdS. The third-order Birch-Murnaghan equation of state given by Eq. 1

$$
P=1.5 B_{0}\left[\left(V / V_{0}\right)^{-\frac{7}{3}}-\left(V / V_{0}\right)^{-\frac{5}{3}}\right] x\left\{1+0.75\left(B_{0}^{\prime}-4\right)\left[\left(V / V_{0}\right)^{-\frac{2}{3}}-1\right]\right\}
$$

was used to fit the energy-volume data for three structures of CdS. Where $P$ is the pressure, $V$ is the volume at pressure, $V o, B o$ and $B_{0}^{\prime}$ are the volume, bulk modulus and its pressure derivate at $0 \mathrm{GPa}$, respectively $[24,25]$. The total energy as a function of volume is depicted 
in Fig. 3. Table 2 reports the equilibrium lattice parameters, equilibrium volume rates, bulk modulus and their pressure derivatives, together with the other theoretical and experimental data for all structures of CdS. It was found that the current theoretical lattice constant and bulk modulus are in good agreement with experimental data within 1-2\% that the choice of generalized gradient approximation for this study strongly supports to other theoretical and experimental results.

We used the Gibbs free energy $(G)$ in order to calculate the phase transition pressures from $\mathrm{WZ}$ to $\mathrm{NaCl}$-type structure and from $\mathrm{NaCl}$ to $\mathrm{CdS}$-III-type structure at $0 \mathrm{~K}$. The Gibbs free energy is given by Eq. 2.

$G=E_{t o t}+P V-T S$

Where $E$ is the total energy, $P$ is the pressure, $V$ is the volume and $S$ is the entropy. We performed our calculations at $0 \mathrm{~K}$, therefore, the $T S$ term is neglected. Thus, $G$ equals to the enthalpy $(H)$ given by Eq. 3.

$H=E_{\text {tot }}+P V$

where $P=-d E_{\text {tot }} / d V$.

The intersection of two enthalpy curves shows a transition pressure between two phases. The phase transition pressures from $\mathrm{WZ}$ to NaCl-type structure is found to be about 3 $\mathrm{GPa}$, and from $\mathrm{NaCl}$ to CdS-III-type structure is found to be about $51 \mathrm{GPa}$, and the related enthalpy versus pressure graphs for the all phases at CdS are depicted in Fig. 4.

As a next step, we studied the atomic movement during the phase transformation by analyzing the modification of the simulation cell. We plot the variation of the simulation cell lengths and angles as a function of minimization step at $10 \mathrm{GPa}$ and $165 \mathrm{GPa}$ in Fig. 5, respectively. These cell vectors are expressed as A, B, and C are initially along the [100], [010] and [001] directions, respectively. The magnitudes of these vectors are plotted in Fig. 5.

In Fig. 5a, there is no change in angles $\alpha$ (between $\mathrm{B}$ and $\mathrm{C}$ lattice vectors), $\beta$ (between $\mathrm{A}$ and $\mathrm{C}$ lattice vectors) and $\gamma$ (between $\mathrm{A}$ and $\mathrm{B}$ lattice vectors) between 0 and $20^{\text {th }}$ minimization steps. However, after $20^{\text {th }}$ minimization step, a dramatic increase is seen in $\gamma$, up to $140^{\circ}$, because of phase transformation from $\mathrm{P}_{3} \mathrm{mc}$ to $\mathrm{Fm} \overline{3} \mathrm{~m}$. All angles do not display a significant change after $55^{\text {th }}$ minimization step. The lattice lengths for $|A|,|B|$ and $|C|$ are 
fairly steady until $25^{\text {th }}$ minimization step. Afterwards, a noticeable increase is seen in $|A|$, whilst a sharp decrease is noticed in $|B|$ and $|C|$.

In Fig. $5 \mathrm{~b}$, there is a dramatic decrease in $\alpha$ angle at around $40^{\text {th }}$ minimization step to $65^{\text {th }}$ minimization step. Afterwards, $\alpha$ angle does not show much change. In the meantime, $\beta$ and $\gamma$ angles stay fairly stable. The lattice lengths of $|B|$ and $|C|$ exhibit a decreasing trend whereas $|A|$ shows an increase in lattice lengths until $60^{\text {th }}$ minimization step. Then all the lattice lengths show a steady trend.

In order to investigate whether there is any intermediate state during the phase transformation or not, we analyzed the structure obtained in that study at each minimization step with KPLOT program. For the NaCl-type structure with space group Fm $\overline{3} \mathrm{~m}$ of CdS, we determine an orthorhombic structure with $\mathrm{Cmc}_{1}$ symmetry at 11 minimization step, a monoclinic structure with $\mathrm{P} 2_{1}$ symmetry at 15 minimization step, another orthorhombic structure with Pmn2 $2_{1}$ symmetry at 33 minimization step, another monoclinic structure with $\mathrm{P} 21 / \mathrm{m}$ symmetry at 35 minimization step, another orthorhombic structure with Pmmn symmetry at 36 minimization step, a tetragonal structure with $14 / \mathrm{mmm}$ symmetry at 44 minimization step and another orthorhombic structure with $\mathrm{Cmcm}$ symmetry at 45 minimization step. The cubic NaCl-structure having $\mathrm{Fm} \overline{3} \mathrm{~m}$ symmetry forms at 53 minimization step.

For the CdS-III- type structure with space group Pmmn of CdS, we determine an orthorhombic structure with Immm symmetry at 10 minimization step and a monoclinic structure with $\mathrm{P} 21 / \mathrm{m}$ symmetry at 34 minimization step. The orthorhombic CdS-III-type structure having Pmmn symmetry forms at 105 minimization step. These intermediary states are depicted in Fig. 6 for evolution of the Fm $\overline{3} \mathrm{~m}$ phase and in Fig. 7 for evolution of the Pmmn phase.

We calculated the electronic band structures (EBS) and corresponding total density of states (DOS) for CdS along the high-symmetry directions and illustrated near the Fermi energy $\left(E_{F}\right)$ level as a function of energy in Fig. 8 and Fig. 9, respectively. Fermi level was set to be $0 \mathrm{eV}$. The symmetry points are $\mathrm{A}, \mathrm{L}, \mathrm{H}, \mathrm{A}, \Gamma, \mathrm{M}, \mathrm{K}$ and $\Gamma$ for $\mathrm{P} 6_{3} \mathrm{mc}$ phase, $\Gamma, \mathrm{X}, \mathrm{W}, \Gamma, \mathrm{L}, \mathrm{W}$ and $\mathrm{L}$ for $\mathrm{Fm} \overline{3} \mathrm{~m}$ phase and $\Gamma, \mathrm{X}, \mathrm{S}, \mathrm{Y}, \Gamma, \mathrm{Z}, \mathrm{U}, \mathrm{R}$ and $\mathrm{T}$ for Pmmn phase. As seen from EBS of CdS, the valance band is located below the $E_{F}$ level, while the conduction band is located above it.

Our results indicate that $\mathrm{P}_{3} \mathrm{mc}$ phase of CdS corresponds to direct band transition with a band gap of $2.28 \mathrm{eV}$. Because the valance band maxima and the conduction band minima lie on the 
same symmetry point $(\Gamma-\Gamma)$. Fm $\overline{3} \mathrm{~m}$ phase of $\mathrm{CdS}$ corresponds to indirect band transition with a band gap of $1.23 \mathrm{eV}$. This appears between $\mathrm{W}$-point (the valence band maxima) and $\Gamma$-point (the conduction band minima). The obtained band gap values for $\mathrm{P} 6_{3} \mathrm{mc}$ and $\mathrm{Fm} \overline{3} \mathrm{~m}$ phases of CdS are compared with other theoretical and experimental values in Table 3.

There are energy gaps $\left(E_{g}\right)$ between valance band and conduction band in $\mathrm{P} 6_{3} \mathrm{mc}$ and $\mathrm{Fm} \overline{3} \mathrm{~m}$ phases of CdS. This suggested that first two phases of CdS indicate semiconductor behavior. Pmmn phase of CdS has metallic character due to the absence of the band gap at Fermi energy level.

We calculated the partial density of states (PDOS) to obtain further information about the electronic nature of CdS and depicted in Fig. 10. It can be seen from Fig. 10 that the largest contribution came from S-3p states at Fermi level for Pmmn phase, from S-3p states between $0 \mathrm{eV}$ and $-6 \mathrm{eV}$ for $\mathrm{Fm} \overline{3} \mathrm{~m}$ and from Cd-5s states between $0 \mathrm{eV}$ and $+8 \mathrm{eV}$ for $\mathrm{Fm} \overline{3} \mathrm{~m}$ phase.

\section{Conclusion}

In summary, the structural and electronic properties of $\mathrm{CdS}$ under high hydrostatic pressure ranging from 0 to $200 \mathrm{GPa}$ at zero temperature have been studied using ab initio constant pressure simulations. We obtained phase transition sequence $\mathrm{P}_{3} \mathrm{mc} \rightarrow \mathrm{Fm} \overline{3} \mathrm{~m} \rightarrow$ Pmmn. We observed seven intermediate states for $\mathrm{P}_{3} \mathrm{mc} \rightarrow \mathrm{Fm} \overline{3} \mathrm{~m}$ transition and two intermediate states for $\mathrm{Fm} \overline{3} \mathrm{~m} \rightarrow$ Pmmn transition. We concluded that these intermediate phases estimated for the first time in this work. Also we studied electronic properties of CdS and obtained band gaps from EBS about $2.28 \mathrm{eV}$ and $1.23 \mathrm{eV}$ for $\mathrm{P}_{3} \mathrm{mc}$ and $\mathrm{Fm} \overline{3} \mathrm{~m}$ phases, respectively. 


\section{References}

[1] B. Wen, R.V.N. Melnik, Appl. Phys. Lett., 92 (2008) 3.

[2] J. Tan, Y. Li, G. Ji, Acta Physica Polonica A, 120 (2011) 501-506.

[3] M. Cardona, G. Harbeke, Physical Review, 137 (1965) A1467.

[4] O. Zakharov, A. Rubio, X. Blase, M.L. Cohen, S.G. Louie, Physical Review B, 50 (1994) 10780-10787.

[5] Y.N. Xu, W.Y. Ching, Physical Review B, 48 (1993) 4335-4351.

[6] S.H. Wei, S.B. Zhang, Physical Review B, 62 (2000) 6944-6947.

[7] X.S. Zhao, J. Schroeder, T.G. Bilodeau, L.G. Hwa, Physical Review B, 40 (1989) $1257-$ 1264.

[8] M.D. Knudson, Y.M. Gupta, A.B. Kunz, Physical Review B, 59 (1999) 11704-11715.

[9] M. Haase, A.P. Alivisatos, Journal of Physical Chemistry, 96 (1992) 6756-6762.

[10] N. Benkhettou, D. Rached, B. Soudini, M. Driz, Physica Status Solidi B-Basic Research, 241 (2004) 101-107.

[11] Y. Li, X. Zhang, H. Li, X. Li, C. Lin, W. Xiao, J. Liu, Journal of Applied Physics, 113 (2013) 083509.

[12] J. Xiao, B. Wen, R. Melnik, Y. Kawazoe, X. Zhang, Physical Chemistry Chemical Physics, 16 (2014) 14899-14904.

[13] V.V. Shchennikov, S.V. Ovsyannikov, physica status solidi (b), 244 (2007) 437-442.

[14] E. Deligoz, K. Colakoglu, Y. Ciftci, Physica B: Condensed Matter, 373 (2006) 124-130.

[15] S. Ouendadji, S. Ghemid, H. Meradji, F.E.H. Hassan, Computational Materials Science, 50 (2011) 1460-1466.

[16] H. Chen, Y. Zhu, B. Wu, Physica B: Condensed Matter, 406 (2011) 4052-4055.

[17] T. Suzuki, T. Yagi, S. Akimoto, T. Kawamura, S. Toyoda, S. Endo, Journal of Applied Physics, 54 (1983) 748-751.

[18] J.P. Perdew, K. Burke, M. Ernzerhof, Physical Review Letters, 77 (1996) 3865-3868.

[19] P. Ordejón, E. Artacho, J.M. Soler, Physical Review B, 53 (1996) R10441-R10444.

[20] N. Troullier, J.L. Martins, Physical Review B, 43 (1991) 1993-2006.

[21] H.J. Monkhorst, J.D. Pack, Physical Review B, 13 (1976) 5188-5192.

[22] R. Hundt, J.C. Schon, A. Hannemann, M. Jansen, Journal of Applied Crystallography, 32 (1999) 413-416. 
[23] A. Hannemann, R. Hundt, J.C. Schon, M. Jansen, Journal of Applied Crystallography, 31 (1998) 922-928.

[24] F. Birch, Physical Review, 71 (1947) 809-824.

[25] F. Murnaghan, Proceedings of the National Academy of Sciences, 30 (1944) 244-247.

[26] K. Wright, J.D. Gale, Physical Review B, 70 (2004) 035211.

[27] R. Nelmes, M. McMahon, Semiconductors and semimetals, 54 (1998) 145-246.

[28] H. Sowa, Solid State Sciences, 7 (2005) 73-78.

[29] A. Phuruangrat, T. Thongtem, S. Thongtem, Materials Letters, 63 (2009) 1538-1541.

[30] K.S. Ramaiah, R.D. Pilkington, A.E. Hill, R.D. Tomlinson, A.K. Bhatnagar, Materials Chemistry and Physics, 68 (2001) 22-30.

[31] L. Zeiri, I. Patla, S. Acharya, Y. Golan, S. Efrima, J. Phys. Chem. C, 111 (2007) 1184311848.

[32] X. Liu, T. Xing, Solid State Communications, 187 (2014) 72-76.

[33] K. Nishidate, T. Sato, Y. Matsukura, M. Baba, M. Hasegawa, Physical Review B, 74 (2006) 035210-1-8.

[34] L. Shi, Y. Qin, J. Hu, Y. Duan, L. Qu, L. Wu, G. Tang, EPL, 106 (2014) 57001-1-6.

[35] B. Batlogg, A. Jayaraman, J.E. Van Cleve, R.G. Maines, Physical Review B, 27 (1983) 3920-3923.

[36] Stanley W.W. Liu and S. Rabii, Physical Review B, 13 (1976) 1675-1680. 
Table I. The equilibrium lattice parameters and the atomic fractional coordinates of the $\mathrm{P}_{3} \mathrm{mc}, \mathrm{Fm} \overline{3} \mathrm{~m}$ and Pmmn phases.

\begin{tabular}{|c|c|c|c|c|c|c|}
\hline Phases & $\mathbf{a}(\AA)$ & b $(\AA)$ & c $(\AA)$ & $\mathbf{x}$ & $\mathbf{y}$ & $\mathbf{z}$ \\
\hline \multirow[t]{4}{*}{$\mathrm{P6}_{3} \mathrm{mc}$} & 4.1720 & 4.1720 & 6.6819 & Cd: 0.3333 & 0.6667 & 0.0000 \\
\hline & & & & Cd: 0.6667 & 0.3333 & 0.5000 \\
\hline & & & & S: 0.3333 & 0.6667 & 0.6200 \\
\hline & & & & S: 0.6667 & 0.3333 & 0.1200 \\
\hline \multirow[t]{8}{*}{ Fm $\overline{3} \mathbf{m}$} & 5.2926 & 5.2926 & 5.2926 & Cd: 0.0000 & 0.0000 & 0.0000 \\
\hline & & & & Cd: 0.0000 & 0.5000 & 0.5000 \\
\hline & & & & Cd: 0.5000 & 0.5000 & 0.0000 \\
\hline & & & & Cd: 0.5000 & 0.0000 & 0.5000 \\
\hline & & & & S: 0.5000 & 0.5000 & 0.5000 \\
\hline & & & & S: 0.5000 & 0.0000 & 0.0000 \\
\hline & & & & S: 0.0000 & 0.0000 & 0.5000 \\
\hline & & & & S: 0.0000 & 0.5000 & 0.0000 \\
\hline \multirow[t]{5}{*}{ Pmmn } & 4.0373 & 4.8686 & 2.8889 & Cd: 0.2500 & 0.2500 & 0.2551 \\
\hline & & & & 500 & 0.7500 & 0.7449 \\
\hline & & & & & 0.7500 & 0.3157 \\
\hline & & & & 0.2500 & 0.2500 & 0.6843 \\
\hline & & & & S: 0.7500 & & \\
\hline
\end{tabular}


Table II. Theoretical ( $\mathrm{T}=0 \mathrm{~K}) \mathrm{CdS}$ lattice parameters for Wurtzite structure (space group, SG: $\mathrm{P}_{3} \mathrm{mc}$ ) and high-pressure phases: Rocksalt structure (SG:Fm $\left.\overline{3} \mathrm{~m}\right)$ and orthorombic structure (SG:Pmmn) with GGA at the corresponding pressure P. a, b, and c are the lattice parameters, $\mathrm{V}$ is the equilibrium volume at the respective pressure, $\mathrm{B}_{0}$ the bulk modulus, $\mathrm{B}_{0}^{\prime}$ the first derivative of the bulk modulus.

\begin{tabular}{lllllllll}
\hline Phases & $\mathbf{P}(\mathbf{G P a})$ & $\mathbf{a}(\AA)$ & $\mathbf{b}(\AA)$ & $\mathbf{c}(\AA)$ & $\mathbf{V}\left(\AA^{\mathbf{3}}\right)$ & $\mathbf{B}_{\mathbf{0}}(\mathbf{G P a})$ & $\mathbf{B}_{\mathbf{0}}^{\prime}$ & References \\
\hline $\mathbf{P 6}_{\mathbf{3}} \mathbf{m c}$ & 0 & 4.1720 & 4.1720 & 6.6819 & 100.72 & 74.85 & 4.22 & This Study \\
& & 4.0860 & 4.0860 & 6.6670 & & 68.90 & 4.70 & {$[26]$} \\
& & 4.1900 & 4.1900 & 6.6600 & 101.42 & 66.40 & & {$[2]$} \\
& & 4.1800 & 4.1800 & 6.7600 & 102.32 & 79.48 & 2.94 & {$[8]$} \\
& & 4.1362 & 4.1362 & 6.7140 & & 99.52 & 4.00 & {$[27]$} \\
& & 4.1380 & 4.1380 & 6.7180 & 99.52 & 62.80 & 4.00 & {$[28]$} \\
Fm $\overline{3} \mathbf{m}$ & 3 & 5.2926 & 5.2926 & 5.2926 & 148.25 & 102.57 & 4.34 & This study \\
& 3 & 5.3210 & 5.3210 & 5.3210 & 157.30 & 105.00 & 4.00 & {$[11]$} \\
& 2.3 & 5.3527 & 5.3527 & 5.3527 & & 97.28 & 4.51 & {$[10]$} \\
& & & & & & & & \\
\hline \multirow{2}{*}{ Pmmn } & 51 & 4.0373 & 4.8686 & 2.8889 & 56.78 & 102.18 & 3.92 & This study \\
& 52.3 & 3.6260 & 4.6210 & 3.2180 & 90.90 & 54.00 & 4.00 & {$[11]$} \\
\hline
\end{tabular}


Table III. Calculated band gaps together with the available theoretical and experimental data for CdS

\begin{tabular}{lcl}
\hline Phases & Band gaps (eV) & References \\
$\mathbf{P 6}_{3} \mathbf{m c}$ & 2.28 & This Study \\
& 2.50 & {$[29]$} \\
& 2.42 & {$[30]$} \\
& 2.50 & {$[31]$} \\
& 1.10 & {$[32]$} \\
& 1.20 & {$[33]$} \\
& 1.47 & {$[34]$} \\
& 2.09 & {$[34]$} \\
$\mathbf{F m} \mathbf{3} \mathbf{m}$ & 1.23 & This Study \\
& 1.70 & {$[35]$} \\
& 1.50 & {$[36]$} \\
\hline
\end{tabular}




\section{FIGURE CAPTIONS}

Figure 1: (Color online) Crystal structures of CdS: at (a) $0 \mathrm{GPa}$, (b) $10 \mathrm{GPa}$ and (c) $165 \mathrm{GPa}$.

Figure 2: (Color online) Volume-pressure and lattice constants-pressure relation of CdS.

Figure 3: (Color online) The energy-volume curves of main phases of CdS.

Figure 4: (Color online) The enthalpy curves of $\mathrm{CdS}$ as function of volume.

Figure 5: (Color online) The simulation cell lengths and angles as a function of the minimization step at (a) $10 \mathrm{GPa}$ and (b) $165 \mathrm{GPa}$.

Figure 6: (Color online) At $10 \mathrm{GPa}$, evolution of the $\mathrm{Fm} \overline{3} \mathrm{~m}$ phase at minimization steps.

Figure 7: (Color online) At $165 \mathrm{GPa}$, evolution of the Pmmn phase at minimization steps.

Figure 8: (Color online) The calculated electronic band structures of CdS: P6 $6_{3}$ mc phase (top), Fm̄̄m phase (middle) and Pmmn phase (bottom).

Figure 9: (Color online) The calculated total density of states for CdS.

Figure 10: (Color online) The calculated partial density of states for CdS. 

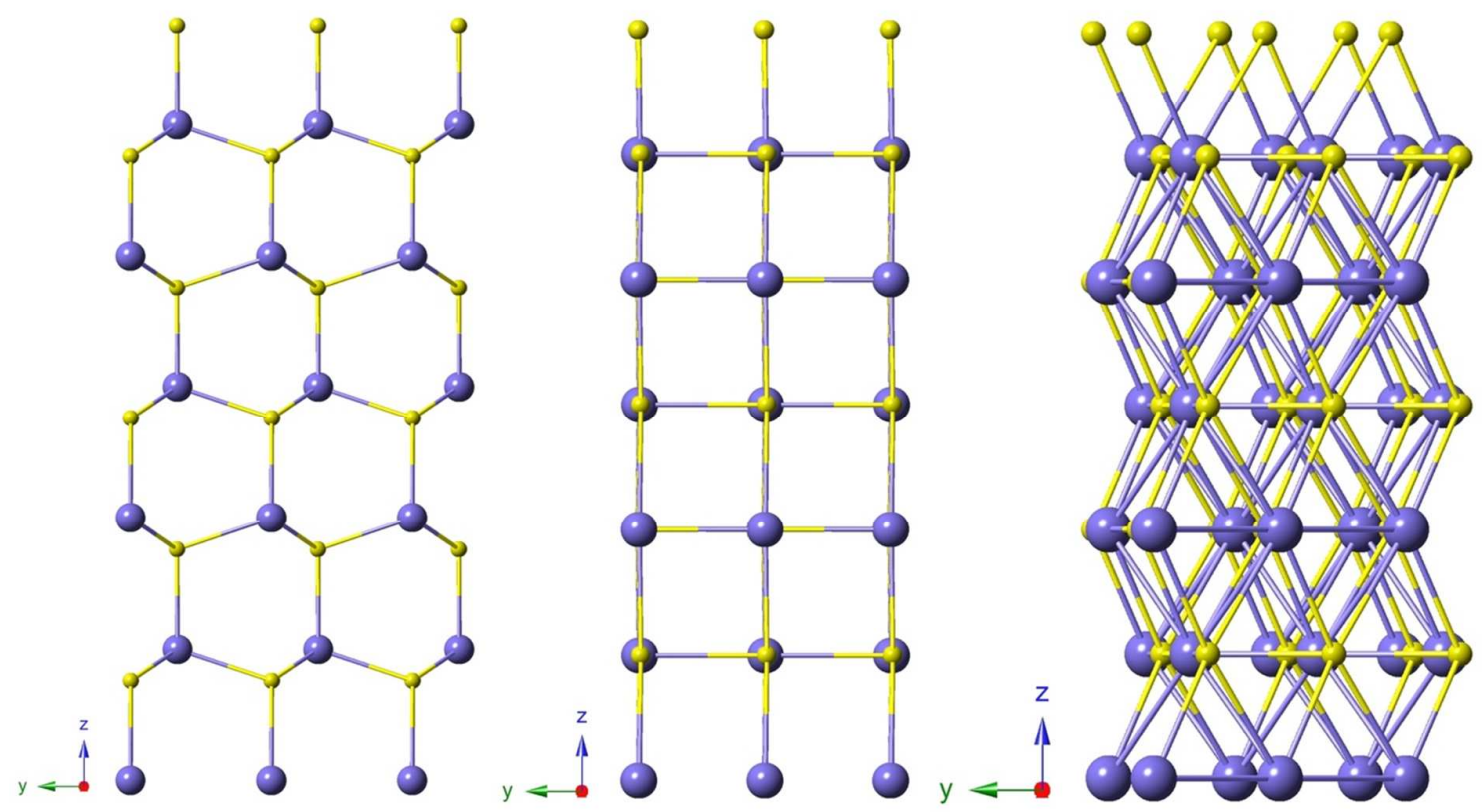

Figure 1 

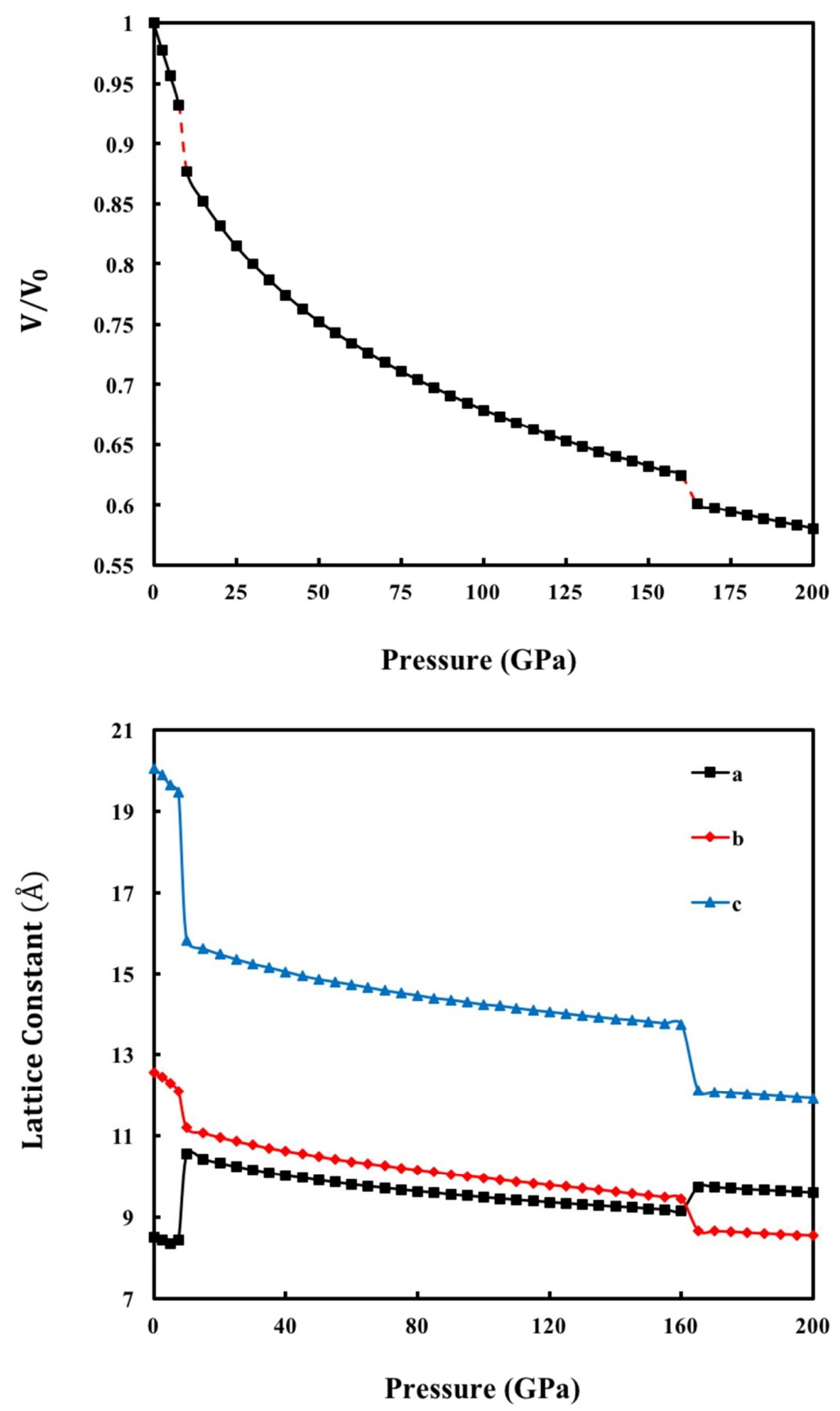

Figure 2 


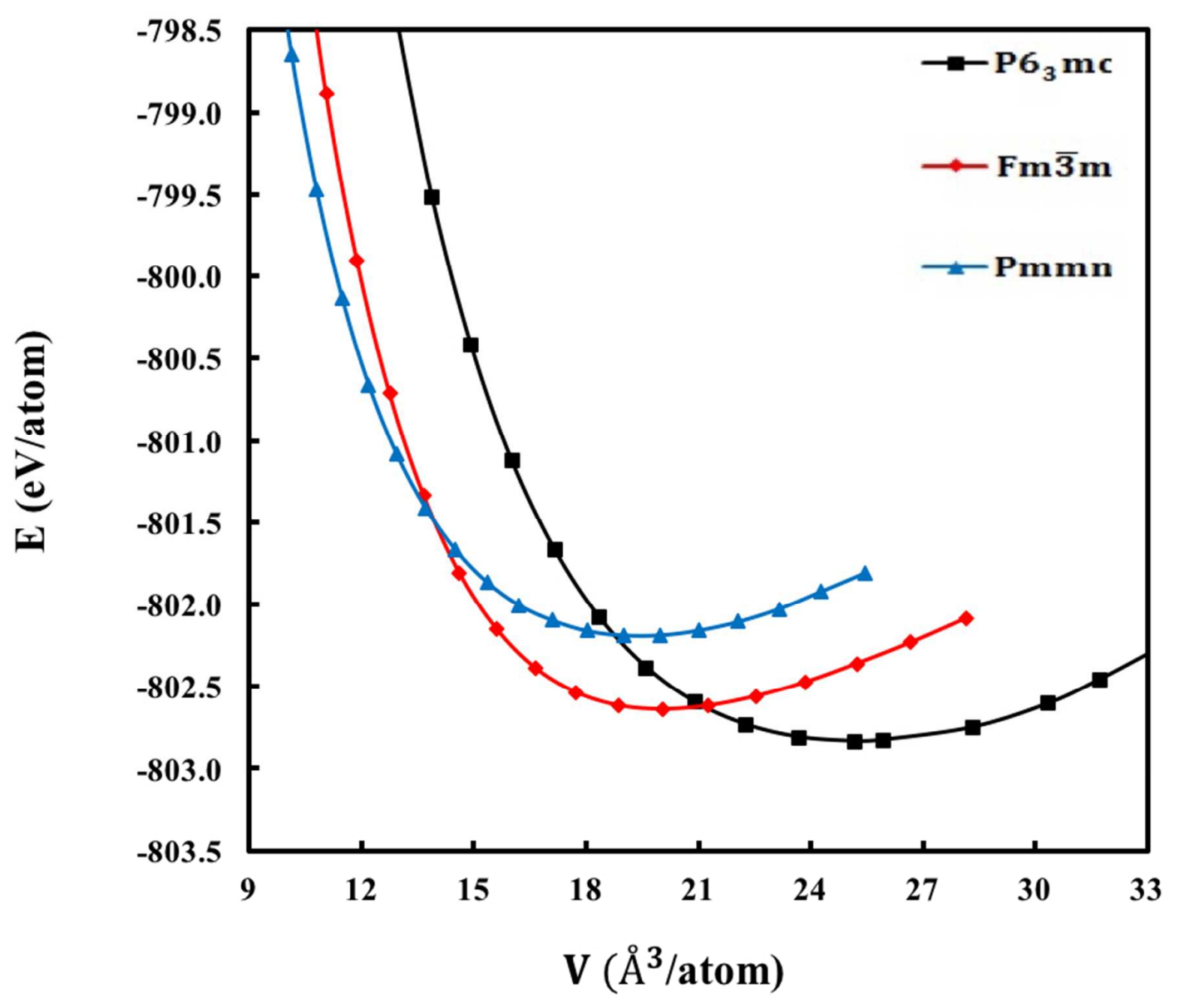

Figure 3 

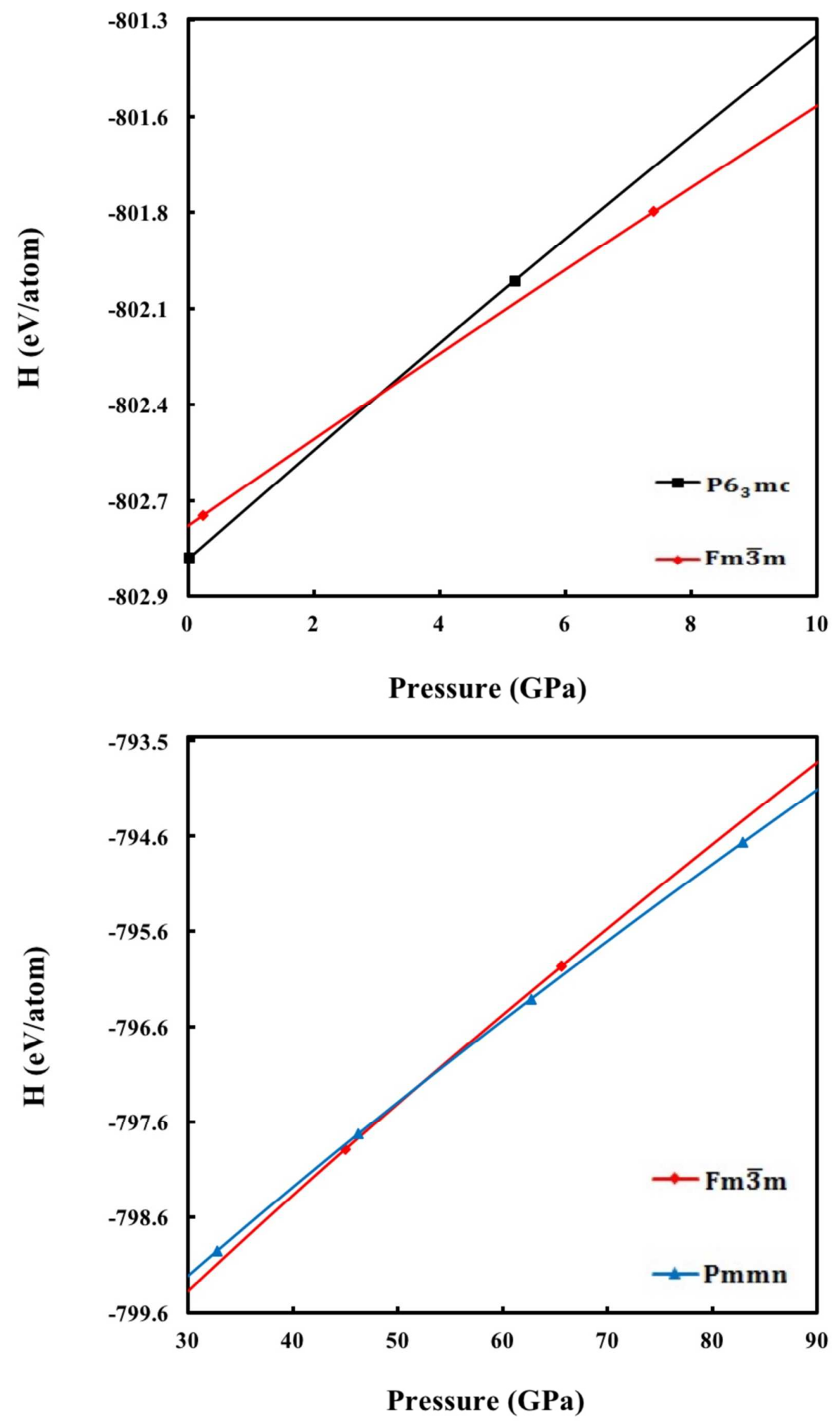
Figure 4
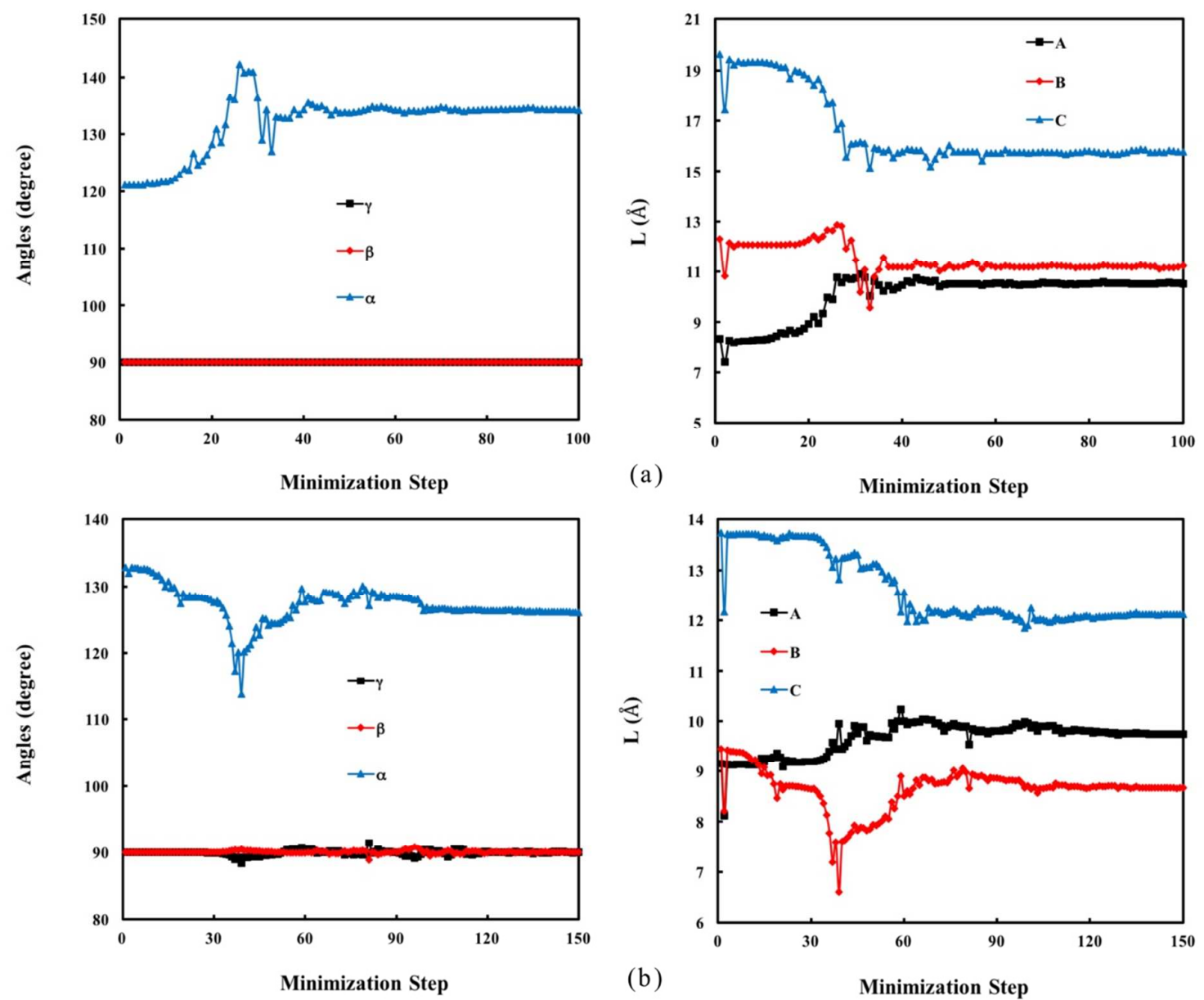

Figure 5 

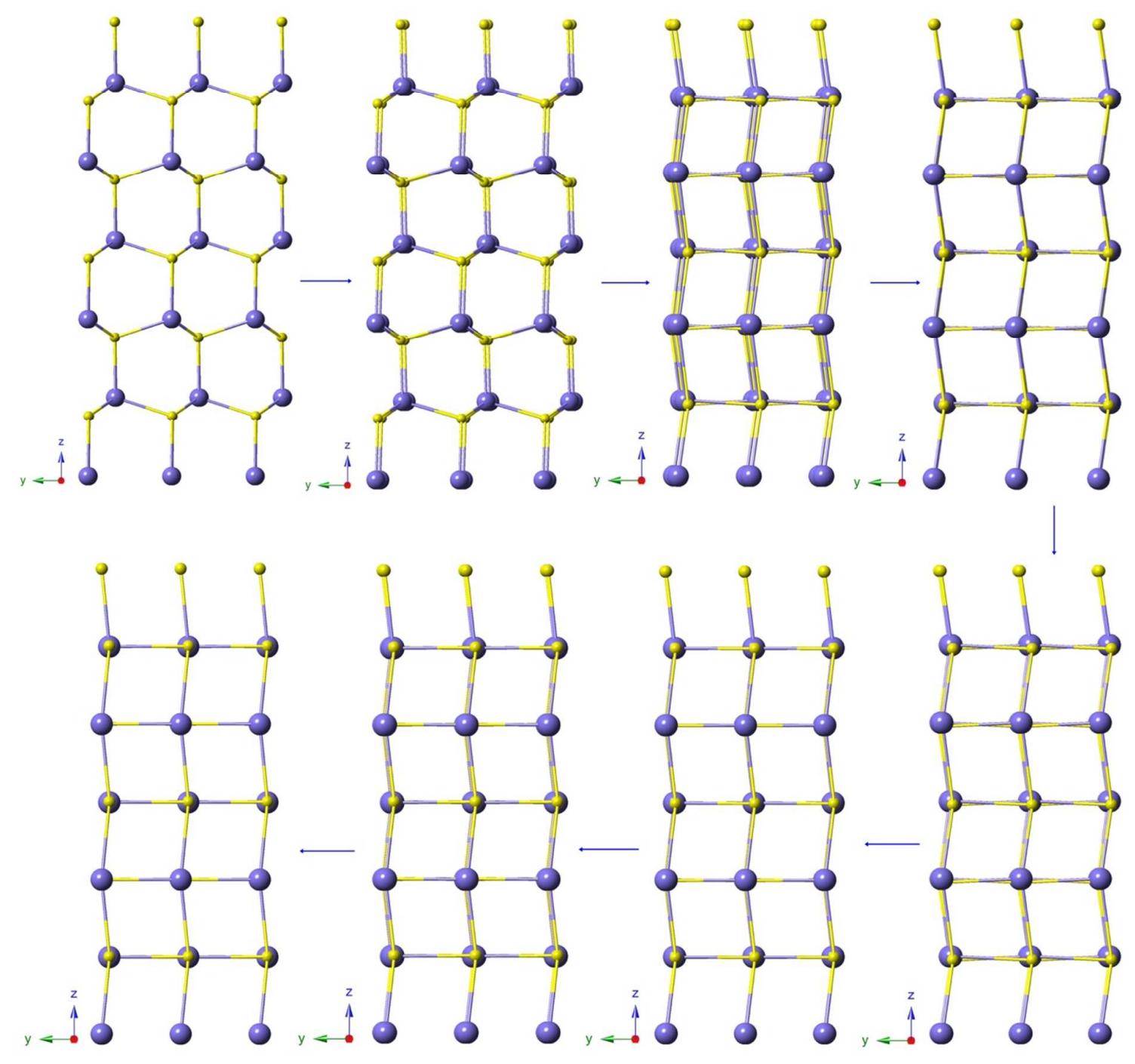

Figure 6 


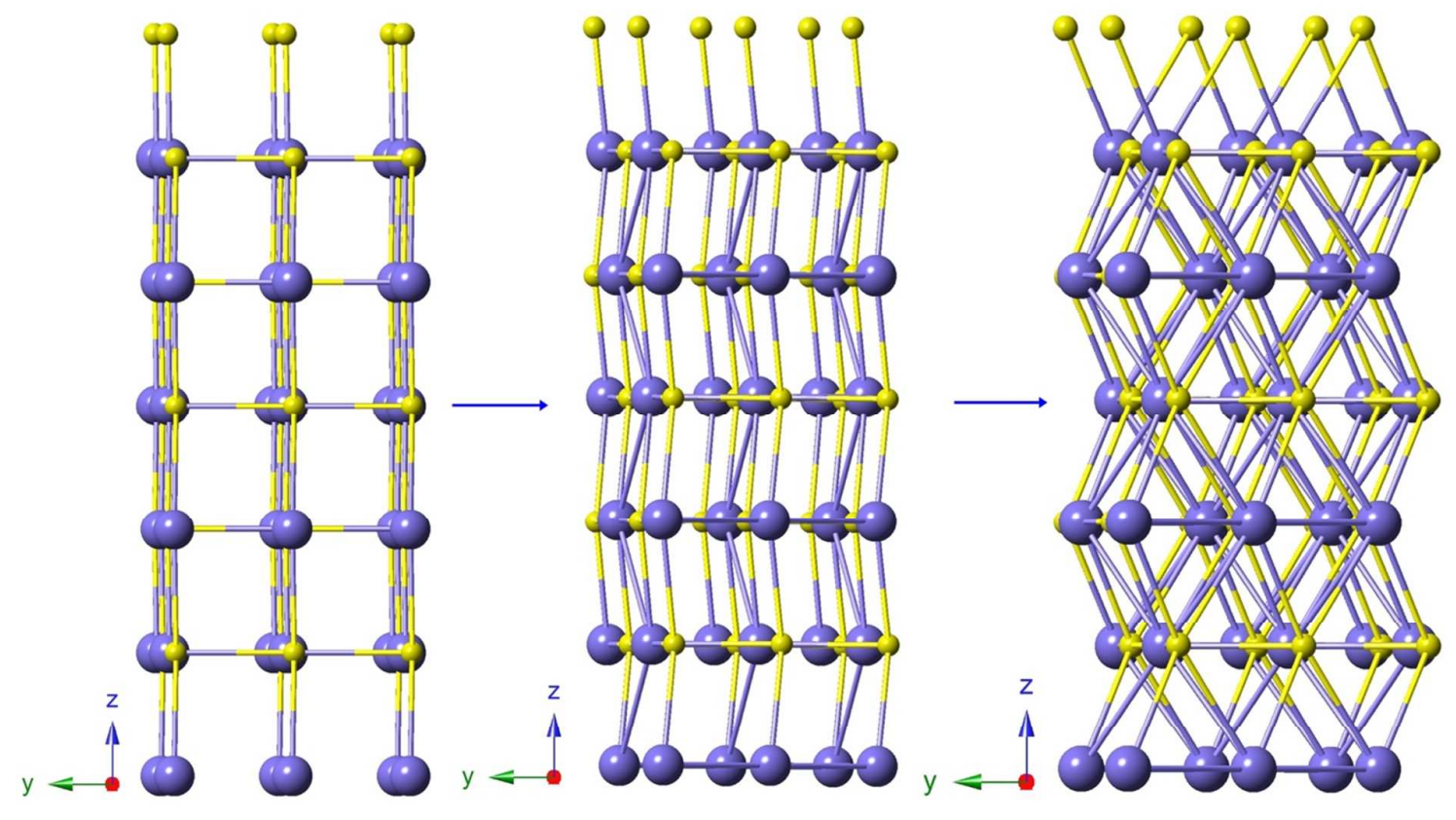

Figure 7 

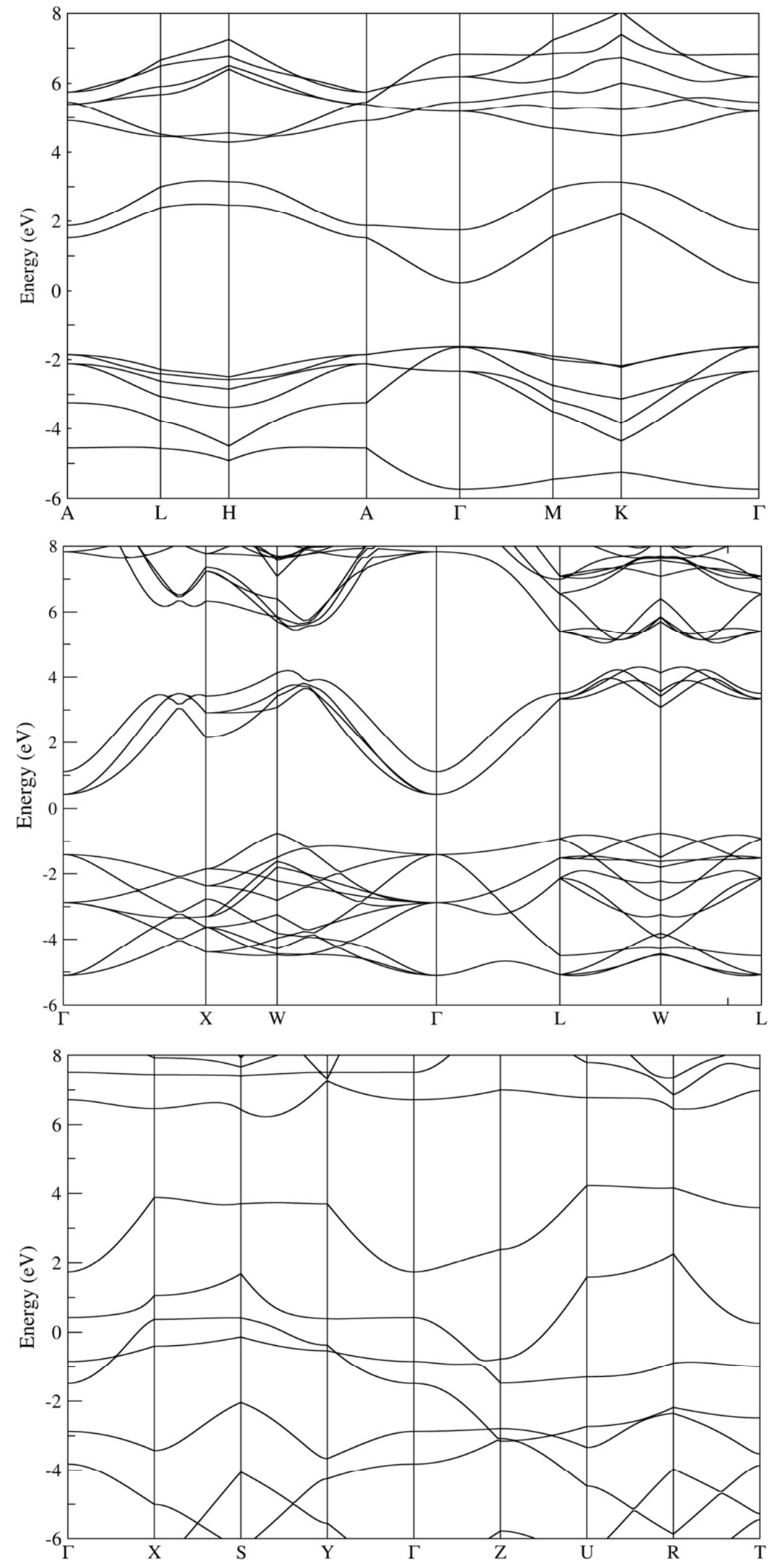

https://mc06.manuscinpftcentral.com/cjp-pubs 
Figure 8

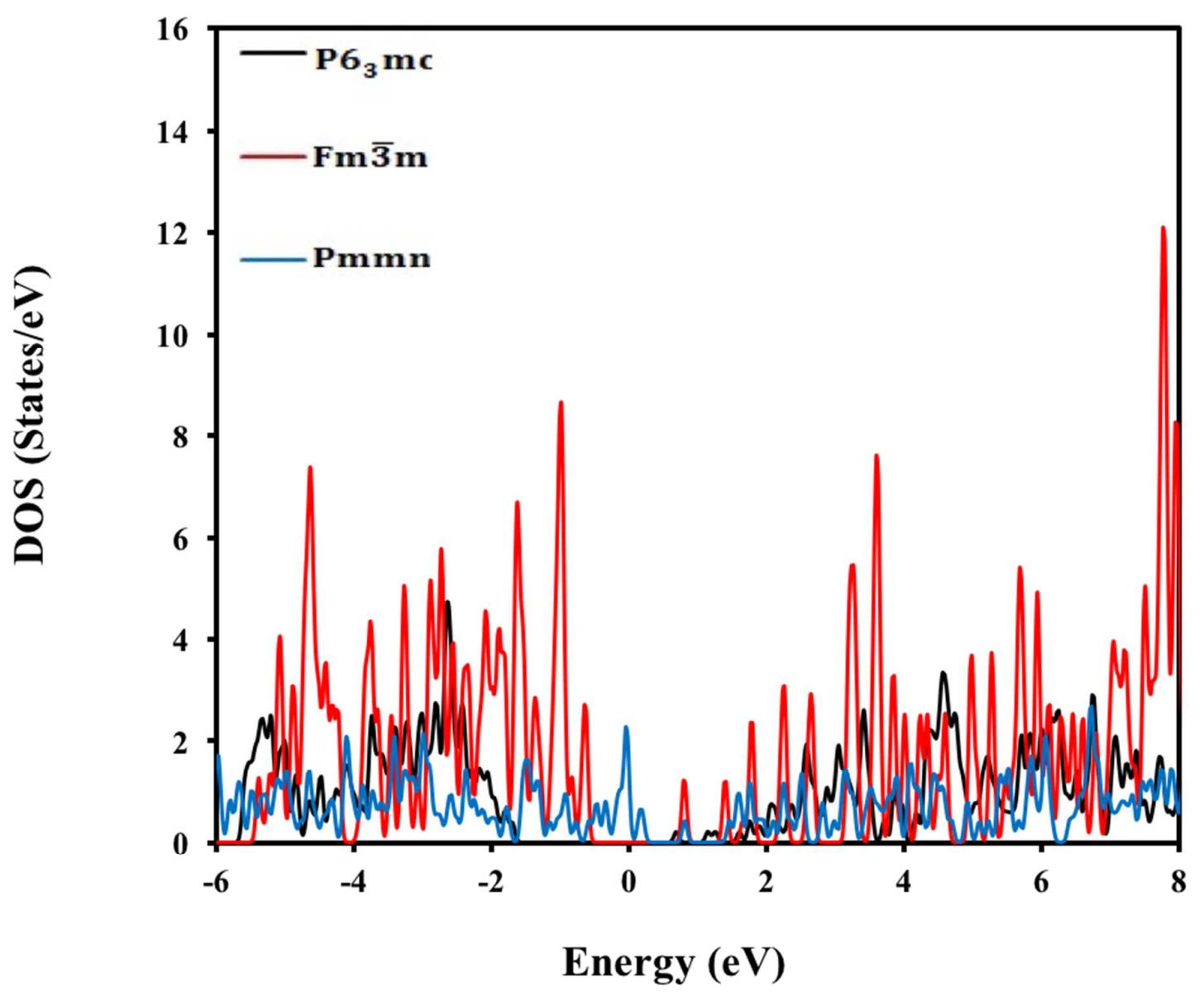

Figure 9 

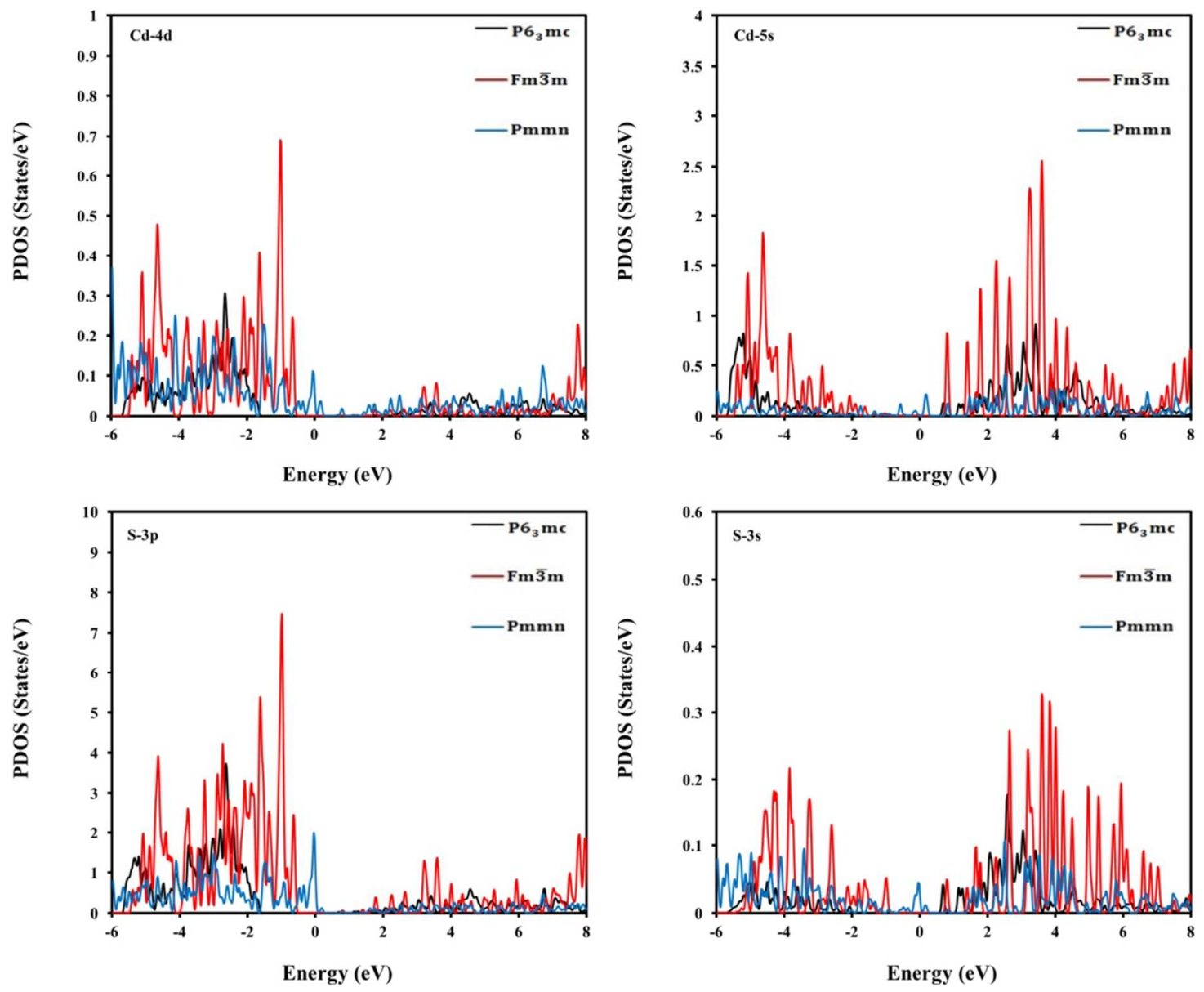

Figure 10 